\title{
Involvement of CD44 in mast cell proliferation during terminal differentiation
}

\author{
Hirotsugu Takano ${ }^{1}$, Shunsuke Nakazawa ${ }^{1}$, Naritoshi Shirata', Shigero Tamba ${ }^{1}$, Kazuyuki Furuta ${ }^{1}$, Sohken Tsuchiya ${ }^{1}$, \\ Kazushi Morimoto', Naoki Itano ${ }^{2}$, Atsushi Irie ${ }^{3}$, Atsushi Ichikawa ${ }^{4}$, Koji Kimata ${ }^{5}$, Kazuhisa Nakayama', \\ Yukihiko Sugimoto ${ }^{1}$ and Satoshi Tanaka ${ }^{4,6}$
}

By using the recently established culture system that reproduces the terminal differentiation process of connective tissuetype mast cells, we found significant transcriptional induction of CD44. As CD44 is a primary receptor for hyaluronan (HA), which is one of the major extracellular matrix components, we investigated the role of CD44 in cutaneous mast cells. When co-cultured with fibroblasts, mouse bone marrow-derived cultured mast cells (BMMCs) were found to form clusters in an HA-dependent manner. As compared with BMMCs derived from the wild-type mice, those from the CD44 ${ }^{-1-}$ mice exhibited impaired growth during the co-cultured period. Furthermore, in the peritoneal cavities and ear tissues, mature mast cells were fewer in number in the $C D 44^{-/-}$mice than in the wild-type mice. We investigated roles of CD44 in mast cell proliferation by reconstituting BMMCs into the tissues of mast cell-deficient, $K i t^{\mathrm{W}} / \mathrm{Kit}^{\mathrm{W}-\mathrm{v}}$ mice, and found that the number of metachromatic cells upon acidic toluidine blue staining in the tissues transplanted with $C D 44^{-/-}$BMMCs was not significantly changed for 10 weeks, whereas that in the tissues transplanted with the CD44 ${ }^{+/+}$BMMCs was significantly increased. These results suggest that CD44 plays a crucial role in the regulation of the cutaneous mast cell number.

Laboratory Investigation (2009) 89, 446-455; doi:10.1038/labinvest.2008.159; published online 9 February 2009

KEYWORDS: CD44; fibroblast; hyaluronan; mast cell; proliferation

Mast cells have the potential to produce diverse arrays of mediators, and release them in the context of the responses, in which local mast cells are involved. ${ }^{1,2}$ Mast cells originate from hematopoietic progenitor cells and undergo terminal differentiation in tissues where they are ultimately resident. ${ }^{3}$ As heterogeneity of tissue mast cells arise as a result of differences in the microenvironment that affects the process of the terminal differentiation, extensive investigation of the maturation process is required for precise understanding of the roles of tissue mast cells. Although several mast cell lines and IL-3-dependent bone marrow-derived cultured mast cells (BMMCs) have been used as models to investigate the process of mast cell activation and subsequent production of proinflammatory mediators, these models have limitations in analyzing functions specific to mature mast cells. Hence, recent studies have often employed the mast cell knock-in models using the mast cell-deficient mice, such as WBB6F1$\mathrm{Kit}^{W} / \mathrm{Kit}^{W-v}\left(\mathrm{Kit}^{W} / \mathrm{Kit}^{W-v}\right)$ and C57BL/6-Kit ${ }^{W-s h} / \mathrm{Kit}^{W-s h}$ $\left(\mathrm{Kit}^{\mathrm{W}-\mathrm{sh}} / \mathrm{Kit}^{\mathrm{W}-\mathrm{sh}}\right)$, to investigate the roles of mature mast cells in vivo. ${ }^{1}$

By modifying the co-culturing method of BMMCs together with fibroblasts in the presence of stem cell factor (SCF), we have recently established a culture system for development of mature mast cells, which exhibit properties characteristic of connective tissue-type mast cells (CTMCs), such as degranulation induced by cationic secretagogues, Safranin-positive granules, and high levels of mast cell protease expression. ${ }^{4}$ We then investigated the gene expression profile during the culture period and identified a cluster of candidate genes, which have potentials to modulate mast cell

\footnotetext{
${ }^{1}$ Department of Physiological Chemistry, Graduate School of Pharmaceutical Sciences, Kyoto University, Kyoto, Japan; ${ }^{2}$ Division of Molecular and Cellular Biology, Department of Molecular Oncology, Institute on Aging and Adaptation, Shinshu University Graduate School of Medicine, Matsumoto, Japan; ${ }^{3}$ Biomembrane Signaling Project, Tokyo Metropolitan Institute of Medical Science, Tokyo, Japan; ${ }^{4}$ Institute for Biosciences, School of Pharmacy and Pharmaceutical Sciences, Mukogawa Women's University, Nishinomiya, Japan; ${ }^{5}$ Research Complex for the Medicine Frontiers, Aichi Medical University, Nagakute, Japan and ${ }^{6}$ Department of Immunobiology, School of Pharmacy and Pharmaceutical Sciences, Mukogawa Women's University, Nishinomiya, Japan

Correspondence: Dr Satoshi Tanaka, PhD, Department of Immunobiology, School of Pharmacy and Pharmaceutical Sciences, Mukogawa Women's University, Kyuban-cho 11-68, Koshien, Nishinomiya, Hyogo 663-8179, Japan.
}

E-mail: s_tanaka@mukogawa-u.ac.jp

Received 4 September 2008; revised 30 October 2008; accepted 4 November 2008 
maturation. Among these genes, whose expression levels are upregulated, we focused on CD44 in the current study.

An array of studies has revealed that CD44 is involved in various cellular responses, such as adhesion to the extracellular matrix, migration, cytokine synthesis, and proliferation..$^{5-7}$ As CD44 is a surface receptor for hyaluronan (HA), which is one of the major components of the extracellular matrix in the cutaneous tissues, ${ }^{8}$ we speculated that CD44 plays some roles in the terminal differentiation of mast cells in the cutaneous tissues. Cutaneous mast cells have recently received much attention as one of the essential immune modulators. For example, TNF- $\alpha$ derived from the cutaneous mast cells was found to be involved in lymph node hypertrophy upon bacterial infection and in the migration of Langerhans cells to the draining lymph nodes in response to bacterial peptidoglycan. ${ }^{9,10}$ Cutaneous mast cells are also crucial for appropriate peripheral tolerance to skin allografts. ${ }^{11}$

In this study, we investigated the roles of CD44, during the terminal differentiation of mast cells. We used two approaches to determine the function of CD44 in mast cells: one is the SCF-dependent co-culture system of BMMCs with fibroblasts and the other is a reconstitution system of mast cells into the $\mathrm{Kit}^{W} / \mathrm{Kit}^{\mathrm{W-v}}$ mice. We show here that CD44 is involved in the proliferation of mast cells during their terminal differentiation in cutaneous tissues.

\section{MATERIALS AND METHODS \\ Mice}

Specific-pathogen-free, 8-week-old female Balb/c mice and 6to 8-week-old male WBB6F1-Kit ${ }^{W} / \mathrm{Kit}^{\mathrm{W}-\mathrm{v}}$ mice were obtained from Japan SLC (Hamamatsu, Japan), and all mice were kept in a specific pathogen-free animal facility at Kyoto University. The $C D 44^{+/-}$mice ${ }^{12}$ backcrossed for 6-10 generations to C57BL/6 were bred to generate the $C D 44^{+/+}$and $C D 44^{-1-}$ mice. All animal experiments were performed according to the Guidelines for Animal Experiments of Kyoto University and were approved by the Committee for Animal Experiments.

\section{Materials}

The following materials were purchased from the sources indicated: hyaluronidase from bovine testis, and an anti-dinitrophenyl (DNP) IgE (clone SPE-7) from Sigma-Aldrich (St Louis, MO, USA); an anti-CD44 antibody (clone KM81) from Cedarlane (Burlington, ON, Canada); an anti-CD44 antibody (clone KM201) from Southern Biotech (Birmingham, AL, USA); a rat $\operatorname{IgG}_{2 \mathrm{a}}$ antibody from BioLegend (San Diego, CA, USA); a horseradish peroxidase-conjugated antimouse IgG and anti-rat IgG antibodies from DAKO (Glostrup, Denmark); an anti-CD16/32 (2.4G2), an FITCconjugated anti-mouse IgE, and a phycoerythrin-conjugated anti-c-kit antibody from BD Biosciences (San Diego, CA, USA); an anti-actin and an FITC-conjugated anti-CD44 antibody (clone KM201) from Chemicon (Temecula, CA,
USA); an RNeasy mini kit from Qiagen (Valencia, CA, USA); M-MLV reverse transcriptase, an Alexa546-conjugated antirat IgG antibody, and 293FT cells from Invitrogen (Carlsbad, CA, USA); LightCycler-Fast Start DNA Master SYBR Green I from Roche Diagnostics (Mannheim, Germany); an ECL kit from GE Healthcare (Uppsala, Sweden); biotinylated HAbinding protein (bHABP); an HA determination kit from Seikagaku Corp. (Tokyo, Japan); FITC-labeled avidin from Vector Laboratories (Burlingame, UK); and $\left[{ }^{3} \mathrm{H}\right]$ thymidine $(20 \mathrm{Ci} / \mathrm{mmol})$ from PerkinElmer (Boston, MA, USA). All other chemicals were commercial products of the reagent grade.

\section{Preparation of BMMCs and Co-Culture with Swiss 3T3 Cells}

Preparation of BMMCs was performed as described elsewhere using $10 \mathrm{ng} / \mathrm{ml} \mathrm{IL-3} \mathrm{instead} \mathrm{of} \mathrm{the} \mathrm{WEHI-conditioned}$ medium. ${ }^{13}$ The method for development of the CTMC-like cultured mast cells was reported earlier. ${ }^{4}$ Briefly, BMMCs were co-cultured with mitomycin C-treated Swiss 3T3 cells in the presence of the recombinant mouse SCF $(100 \mathrm{ng} / \mathrm{ml})$. The subculture was performed every 4 days. Overall $>80 \%$ of the viable mast cells were confirmed to be mature mast cells, as assessed by staining with Safranin-O at day 16 .

\section{Quantitative RT-PCR Analysis}

Total RNAs were isolated from the cultured mast cells using an RNeasy Mini Kit, according to the manufacturer's instruction. Total RNAs were reverse-transcribed using the M-MLV reverse transcriptase and amplified by PCR using a primer offset for GAPDH or CD44 as follows: GAPDH, 5'TGAACGGGAAGCTCACTGG- $3^{\prime}$ (forward) and $5^{\prime}$-TCCAC CACCCTGTTGCTGTA-3' (reverse); and CD44, $5^{\prime}$-TTGG GGACTTTGCCTC-3' (forward) and 5'-CAGATAGCGTTG GGATG-3' (reverse).

\section{Immunoblot Analysis}

Immunoblot analysis was performed as described earlier. ${ }^{13}$ An antibody against actin (1:1000) or CD44 (1:200, clone KM201) was used as the primary antibody.

\section{Flow Cytometry}

Mast cells in the ear tissues were enriched as described earlier. ${ }^{14}$ The flow cytometric analysis was performed as described earlier with FACSCalibur (Becton Dickinson, Franklin Lakes, NJ, USA) equipped with the CELLQUEST software. ${ }^{15}$ The dead cell population was gated out by propidium iodide staining.

\section{Immunofluorescence Staining}

The cultured cells were gently rinsed with PBS, fixed with $2 \%$ paraformaldehyde, and incubated with the biotinylated HAbinding protein $(5 \mu \mathrm{g} / \mathrm{ml}, \mathrm{bHABP})$ and an anti-CD44 antibody (KM81, 1:500) in the presence of $1 \%$ bovine serum albumin overnight at $4{ }^{\circ} \mathrm{C}$. The slides were washed and in- 
cubated with FITC-labeled avidin (1:500) and an Alexa546conjugated anti-rat IgG antibody (1:1000) for $1 \mathrm{~h}$. The fluorescent images were obtained using a fluorescence microscope Nikon Eclipse TE300 (Nikon, Tokyo, Japan) and analyzed using the IPLab 3.5.5/J software. The differential interference contrast microscopy (DIC) images were obtained using the same system. The confocal images were obtained with a confocal laser scanning microscope (LSM5 Pascal; Carl Zeiss, Oberkochen, Germany) with C-Apochromat $\times 63 / 1.2$ and analyzed using the LSM5 Pascal software.

\section{Measurement of HA}

The concentrations of HA in the medium were determined using the ELISA-like system for HA with bHABP, according to the manufacturer's instruction.

\section{Measurement of Histamine}

Mast cells were lysed in PBS containing $2 \mathrm{M} \mathrm{NaCl}$ and $0.5 \%$ Triton X-100 at $4{ }^{\circ} \mathrm{C}$. The lysate of mast cells and the ear tissues were centrifuged at $10000 \mathrm{~g}$ for $30 \mathrm{~min}$ at $4{ }^{\circ} \mathrm{C}$. The resultant supernatant was subjected to the assays for histamine as described earlier. ${ }^{13}$

\section{Cell Proliferation Assay}

Cell proliferation was evaluated by measuring $\left[{ }^{3} \mathrm{H}\right]$ thymidine incorporation for $4 \mathrm{~h}$. $\left[{ }^{3} \mathrm{H}\right]$ thymidine $(20 \mathrm{Ci} / \mathrm{mmol})$ was added to the culture $20 \mathrm{~h}$ after the addition of IL-3 or SCF. In case of the measurement of the cell growth of co-cultured cells, the cells were starved for SCF for $6 \mathrm{~h}$ and then cultured in the presence of SCF and $\left[{ }^{3} \mathrm{H}\right]$ thymidine for $4 \mathrm{~h}$. The cellular radioactivity was measured by a liquid scintillation counter.

\section{Counting of the Tissue Mast Cells}

Thin ear sections $(7 \mu \mathrm{m})$ were fixed with Carnoy's solution followed by staining with acidic toluidine blue in $0.6 \mathrm{~N} \mathrm{HCl}$ for $5 \mathrm{~s}$. The number of metachromatic cells was counted.

\section{Mast Cell Reconstitution in the Tissues of the $\mathbf{W} / \mathbf{W}^{\mathbf{v}}$ Mice} BMMCs were injected into the peritoneal cavity $\left(2 \times 10^{6}\right.$ cells/mouse $)$ or ear cutaneous tissues $\left(1 \times 10^{6}\right.$ cells/ear $)$ of the $\mathrm{Kit}^{\mathrm{W}} / \mathrm{Kit}^{\mathrm{W-v}}$ mice.

\section{Statistical Analysis}

Data are presented as the means \pm s.e.m. Statistical significance for comparisons between groups was determined using Student's paired $t$-test or ANOVA. Additional comparisons were made with Dunnet's multiple comparison test for comparison with the control groups or the Tukey-Kramer multiple comparison test for all pairs of column comparison.

\section{RESULTS \\ Induction of CD44 in Mast Cells Co-Cultured with Fibroblasts}

Transcriptional upregulation of CD44 was confirmed in BMMCs during the co-culture with Swiss 3T3 fibroblasts in the presence of SCF (Figure 1a). The CD44 induction was also confirmed at both the protein level and the cell surface expression level (Figure $1 \mathrm{~b}$ and c). Although CD44 protein was also detected in fibroblasts, their expression levels were much lower than those in mast cells during the co-culture period (Figure $1 \mathrm{~b}$ and data not shown).

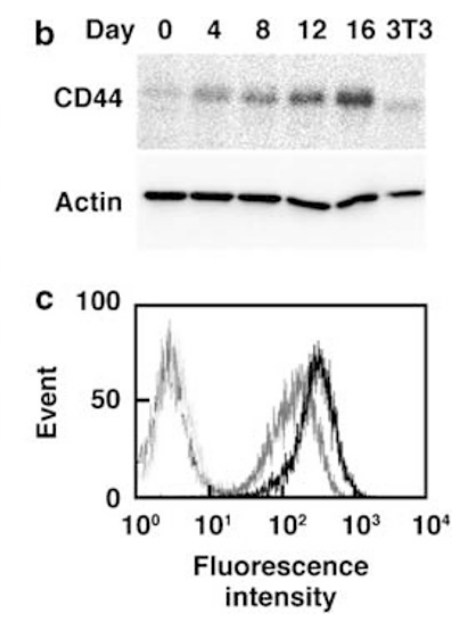

Figure 1 Induction of CD44 during co-culture with Swiss 3T3 fibroblasts. (a) Expression of CD44 mRNA in the co-cultured mast cells was evaluated by quantitative RT-PCR. The expression levels were normalized using those of GAPDH. The relative expression levels were presented as the fold expression. Values are presented as the means \pm s.e.m. of three independent experiments. (b) Expression of CD44 at the protein levels in the co-cultured mast cells and Swiss $3 \mathrm{~T} 3$ fibroblasts was determined by immunoblot analysis. (c) Surface expression of CD44 at day 0 (gray) and day 16 (black) was measured by flow cytometry with an anti-CD44 antibody (KM201, solid lines) and its isotype control antibody (dotted lines).

Figure 2 HA-dependent cluster formation of the co-cultured mast cells. (a) A differential interference contrast microscopy (DIC) image of the co-cultured BMMCs at day 4 is presented. Bar $=100 \mu \mathrm{m}$. (b) Co-cultured cells at day 4 was treated with (HAse) or without (None) hyaluronidase. DIC images were obtained $5 \mathrm{~min}$ after the treatment. Bar $=100 \mu \mathrm{m}$. (c-h) The cells at day 4 in the co-culture system were fluorescently stained with an anti-CD 44 antibody (c and f, red, KM81) and a biotinylated HA-binding protein (d and $\mathbf{g}$, green). DIC images of the same fields were also obtained (e, the same eye field with c and $\mathbf{~ d ) . ~ B a r ~}=200 \mu \mathrm{m}$. The cells stained as described above were observed using the confocal laser-scanning microscopy (f-h). Bar $=50 \mu \mathrm{m}$. (i) The culture medium of Swiss 3T3 cells cultured alone (None) or together with BMMCs (co-culture) was recovered at day 4 and subjected to assay for HA concentration, which was also measured in the case that BMMCs were cultured in the presence of $10 \mathrm{ng} / \mathrm{ml} \mathrm{IL-3} \mathrm{(IL-3)} \mathrm{or} 100 \mathrm{ng} / \mathrm{ml} \mathrm{SCF} \mathrm{(SCF).} \mathrm{Values} \mathrm{are} \mathrm{presented} \mathrm{as} \mathrm{the}$ means \pm s.e.m. from at least three independent experiments. 

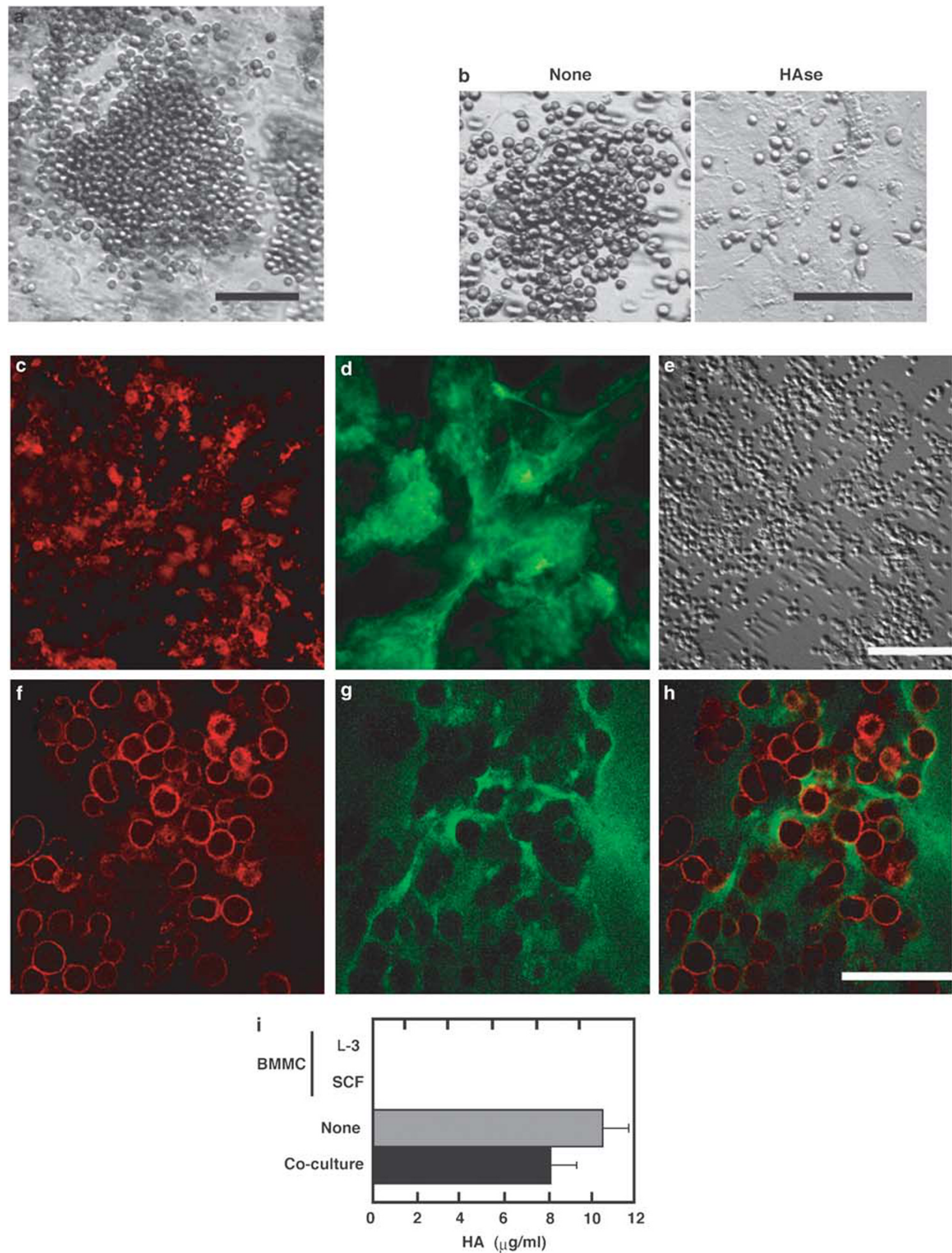


\section{HA-Dependent Cluster Formation of the Cultured Mast Cells}

During the co-culture period, the majority of BMMCs were found to form clusters, which appeared around the third day after every subculture (Figure 2a). As Swiss 3T3 fibroblasts were reported to produce a set of extracellular matrix components including $\mathrm{HA},{ }^{16}$ we speculated that $\mathrm{HA}$ is involved in the formation of these clusters. As expected, these clusters were rapidly and completely disrupted upon treatment with hyaluronidase (Figure $2 \mathrm{~b}$ ), indicating the essential role of HA in the cluster formation. Visualization of HA in the co-culture system using the biotinylated HA-binding protein (bHABP) revealed that HA formed fibrous networks, which developed in close proximity to the mast cells, which were immunoreactive to an anti-CD44 antibody (Figure $2 \mathrm{c}$ and d). Confocal microscopy showed that the HA network was bound to mast cells (Figure $2 \mathrm{f}-\mathrm{h}$ ). Three-dimensional images revealed that the cluster was formed by the connection of individual mast cells by the extracellular matrix containing $\mathrm{HA}$, and that the majority of mast cells were not homotypically aggregated (Supplementary Material 1). Although fluorescent dye-conjugated avidin was found to bind to mast cell granules, ${ }^{17}$ no detectable signals were found in the experiments performed without bHABP (data not shown). We then identified the cells that produce HA in the co-culture system. A significant level of HA was detected in the day 4 coculture medium and comparable to that in the medium of the single culture of Swiss 3T3 fibroblasts (Figure 2i). As HA was not detectable in the culture medium of BMMCs, we concluded that HA in the medium of the co-culture was produced exclusively by fibroblasts.

\section{Impaired Proliferation of CD44 ${ }^{-1-}$ BMMCs in the Co-Culture System}

To determine the role of CD44 expressed in mast cells, we developed BMMCs from bone marrow cells of the CD44 ${ }^{-1-}$ mice and performed the co-culture experiments. There are no differences between $C D 44^{+1+}$ and $C D 44^{-1-}$ BMMCs in size, histamine content, or metachromasy of the acidic Toluidine blue staining (Figure $3 \mathrm{a}$ and data not shown). Furthermore, no significant difference was observed between $\mathrm{CD} 44^{+/+}$and $\mathrm{CD} 44^{-1-}$ BMMCs in the surface expression of Fc $\varepsilon$ RI or c-kit, both of which are typical surface markers of mast cells (Figure 3b). The CD44 deficiency did not change the $\left[{ }^{3} \mathrm{H}\right]$ thymidine uptake and proliferation of BMMCs in response to IL-3 or SCF (Figure $3 \mathrm{c}$ and data not shown). These results indicate that commitment to mast cell lineage and cytokine-induced proliferative response are independent of CD44 in BMMCs, even although they express a low but detectable level of CD44.

In the co-culture system, the growth of $C D 44^{-/-}$mast cells was considerably impaired, in particular at the late phase (Figure 3d). In contrast to the continuous growth of control mast cells, the number of $C D 44^{-1-}$ mast cells leveled off at day 8. In accord with this impaired growth, $\left[{ }^{3} \mathrm{H}\right]$ thymidine uptake during the co-culture period was also decreased in $C D 44^{-1-}$ mast cells (Figure 3e). On the contrary, granule maturation (Figure 3a, lower panels), compound 48/80induced degranulation, or chymotryptic activity of mast cells (Supplementary Figure 1) was not affected by the CD44 deficiency, although the histamine content, tryptic activity, and carboxypeptidase A activity had the tendency to be augmented in $C D 44^{-/-}$mast cells (Supplementary Figure 1). These results suggested that CD44 supports the continuous growth of co-cultured mast cells, but has no or little influence on the phenotypic changes during the maturation period.

\section{Effects of an Anti-CD44 Antibody on the Cluster Size and the Proliferation of Cultured Mast Cells}

A series of studies have been performed using specific antibodies to determine the role of CD44. We investigated the levels of maturation and proliferation of the cultured mast cells in the presence of an anti-CD44 antibody, KM81, which was reported to block HA binding to CD $44 .{ }^{18}$ Proliferation of the co-cultured mast cells was significantly suppressed in the presence of KM81 (Figure 3f). On the other hand, KM81 did not affect the maturation indexes of mast cells, such as the Safranin-O staining, compound 48/80-induced degranulation, histamine content, or granule protease activities (Supplementary Figure 2). These results are consistent with the results obtained using $C D 44^{-/-}$-cultured mast cells.

Treatment with KM81 did not affect proliferation of CD44 ${ }^{-1-}$ mast cells (Figure 3g), which were found to form only small clusters, the size or number of which was not changed in the presence of KM81 (Figure 3h). By contrast, the cluster size of $\mathrm{CD} 44^{+/+}$cultured mast cells was reduced in the presence of KM81. Although co-cultured fibroblasts also express a low level of CD44, these results indicate that the primary target of KM81 is the CD44 expressed in mast cells.

\section{Decrease in the Mast Cell Number in the CD44 ${ }^{-1-}$ Mice} We then compared the two different CTMC populations, peritoneal and cutaneous mast cells, in the $C D 44^{+/+}$and $C D 44^{-1-}$ mice. Flow cytometric analyses confirmed that the c-kit-positive cells in the peritoneal cavities and ear tissues of $\mathrm{CD} 44^{+/+}$mice expressed not only FceRI but also CD44 (Figure 4a), indicating that the mast cell population from these tissues express CD44. The expression levels of FceRI and c-kit were comparable between the $C D 44^{+/+}$and $C D 44^{-1-}$ mast cells (Figure 4a). The results of acidic toluidine blue staining indicated that the size and morphology of tissue mast cells were indistinguishable between $C D 44^{+1+}$ and $C D 44^{-1-}$ mice (Figure $4 \mathrm{~b}-\mathrm{e}$ ). In contrast, both the number and the histamine content of mast cells were significantly lower in the $C D 44^{-1-}$ mice than those in the wildtype mice (Figure $4 \mathrm{f}-\mathrm{i}$ ). The decrease in the number of tissue 
a

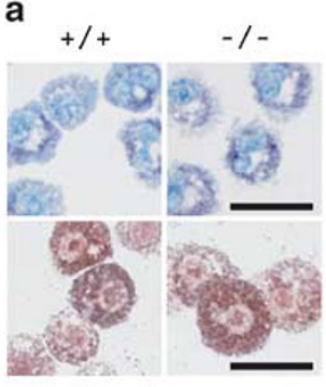

b

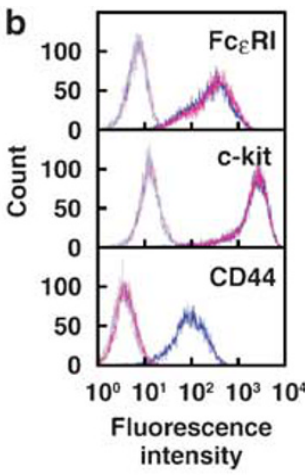

C

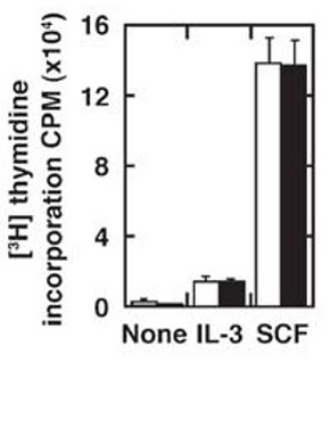

d

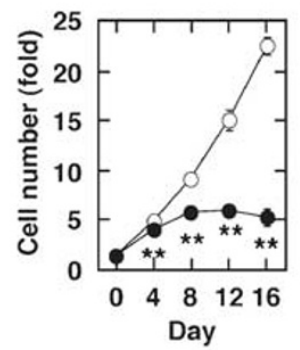

e

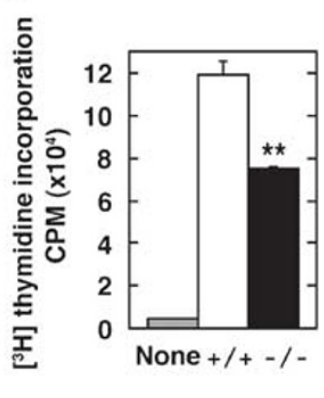

f

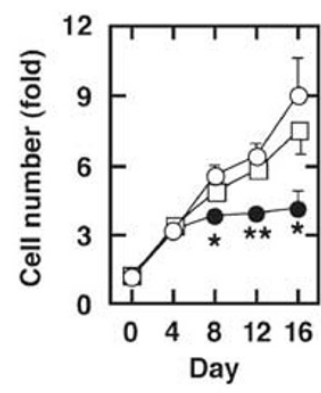

g h

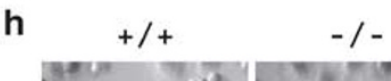

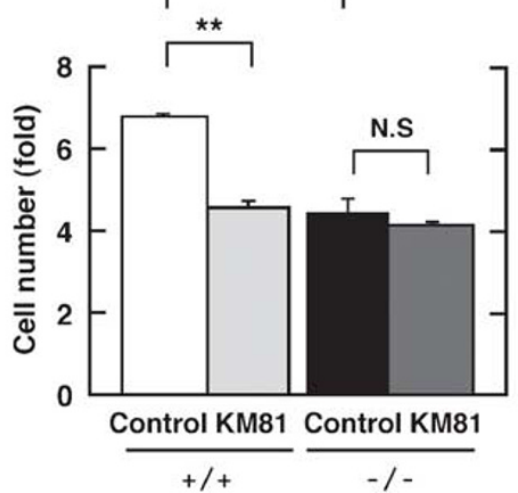

Figure 3 Involvement of CD44 in proliferation of the cultured mast cells. (a) Upper panels show the results of acidic toluidine blue staining of BMMCs derived from the $\mathrm{CD}_{4} 4^{+/+}$and $\mathrm{CD} 44^{-/-}$mice. Lower panels show the results of Alcian blue/Safranin-O staining of the cultured mast cells at day 16.

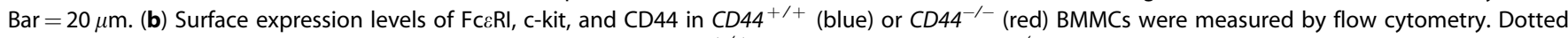
lines show the profiles obtained using the isotype controls. (c) $\mathrm{CD} 44^{+/+}$(open columns) or $C D 44^{-/-}$(closed columns) BMMCs were stimulated without (None) or with $10 \mathrm{ng} / \mathrm{ml} \mathrm{IL-3} \mathrm{(IL-3)} \mathrm{or} 100 \mathrm{ng} / \mathrm{ml} \mathrm{SCF}$ (SCF). Proliferation of the cells were evaluated by the $\left.{ }^{3} \mathrm{H}\right]$ thymidine uptake. Values were presented by the means \pm s.e.m. of three independent experiments. (d) Profiles of the proliferation of $C D 44^{+/+}$(open circle) and $C D 44^{-1-}$ (closed circle) mast cells during the co-culture period are presented. Values (the fold increase in the cell number) are presented as the means \pm s.e.m. of three independent experiments. ${ }^{*} \mathrm{P}<0.01$ vs $\mathrm{CD} 44^{+/+}$mast cells. (e) The $\left[{ }^{3} \mathrm{H}\right]$ thymidine uptake was measured in mitomycin-treated $3 \mathrm{~T} 3$ cells alone (None), co-culture with $C D 44^{+/+}$mast cells $(+/+)$, and co-culture with $C D 44^{-/-}$mast cells $(-/-)$. Values were presented by the means \pm s.e.m. of three independent experiments. ${ }^{*} P<0.01$ vs the co-culture with $\mathrm{CD} 44^{+/+}$mast cells. (f) The $\mathrm{CD} 44^{+/+}$BMMCs were co-cultured in the absence (open squares) or in the presence of an anti-CD44 antibody $(5 \mu \mathrm{g} / \mathrm{ml}$, closed circles, KM81) or its isotype control (open circles). The numbers of the co-cultured mast cells are presented. Values (the fold increase in the cell number) are presented as the means \pm s.e.m. ${ }^{*} P<0.01$ and ${ }^{*} P<0.05$ vs the isotype control. (g and $\mathbf{h}$ ) The number of the $C D 44^{+/+}$and $\mathrm{CD} 44^{-1-}$ co-cultured mast cells at day 8 were compared in the presence of the anti-CD44 antibody $(5 \mu \mathrm{g} / \mathrm{ml}, \mathrm{KM} 81)$ or its isotype control (Control). Values are presented as the means \pm s.e.m. of the three independent experiments. ${ }^{*} P<0.01$ between two columns indicated. NS, not significant. DIC images were obtained at day 4 (h). Bar $=100 \mu \mathrm{m}$.

mast cells in the $C D 44^{-/-}$mice is consistent with the impaired growth of $C D 44^{-/-}$mast cells co-cultured with fibroblasts.

\section{Requirement of CD44 for In Vivo Proliferation of the Mast Cells Reconstituted into Cutaneous Tissues of the $\mathrm{Kit}^{\mathrm{W}} / \mathrm{Kit}^{\mathrm{W}-\mathrm{v}}$ Mice}

As accumulating evidence has indicated that CD44 is involved in infiltration and chemotaxis of a variety of leukocytes, it remained ambiguous whether the decrease in the number of tissue mast cells in the $C D 44^{-/-}$mice resulted from the impaired cell growth or the defect in the migration of immature mast cells. We, therefore, investigated the proliferation of immature mast cells using an in vivo model system, in which BMMCs are reconstituted into the
$\mathrm{Kit}^{W} / \mathrm{Kit}^{\mathrm{W}-\mathrm{v}}$ mice. BMMCs derived from the $\mathrm{CD} 44^{+/+}$or $C D 44^{-1-}$ littermates were injected into the peritoneal cavities or ear tissues of the $\mathrm{Kit}^{W} / \mathrm{Kit}^{W-v}$ mice, and the number and morphology of the transplanted BMMCs were examined 5 or 10 weeks after the initial injection. The transplanted BMMCs derived from $C D 44^{+/+}$and $C D 44^{-1-}$ mice exhibited similar metachromasy in the peritoneal cavities and ear tissues (Figure 5a). In the peritoneal cavities, there was no difference in the number or histamine content of the transplanted mast cells derived from $\mathrm{CD} 44^{+/+}$and $\mathrm{CD} 44^{-/-}$mice (Figure 5b and $\mathrm{c}$ ). On the other hand, the number of metachromatic cells upon toluidine blue staining in the tissues transplanted with $C D 44^{+/+}$BMMCs was significantly increased from 5 to 10 weeks after the initial injection, whereas that in the tissues transplanted with $C D 44^{--}$mast cells was unchanged 
$+1+$

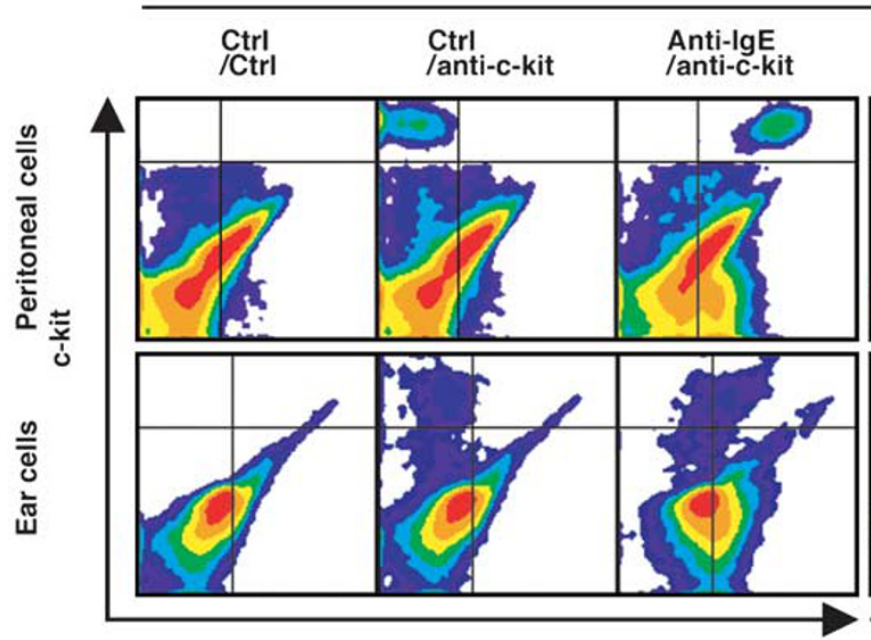

$\mathrm{Fc}_{\varepsilon} \mathrm{RI}$ b

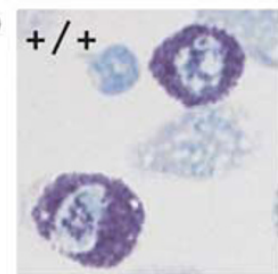

c
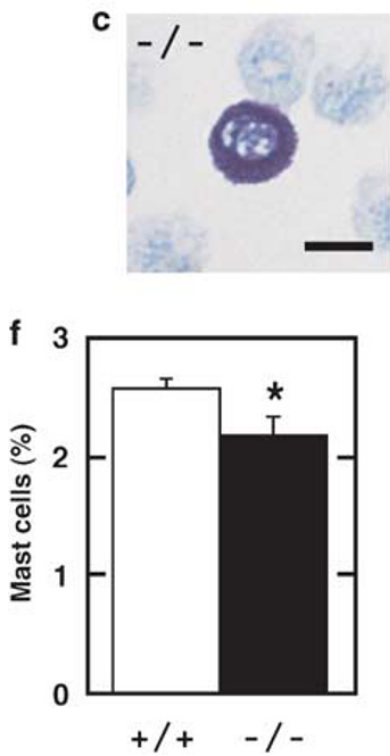

d

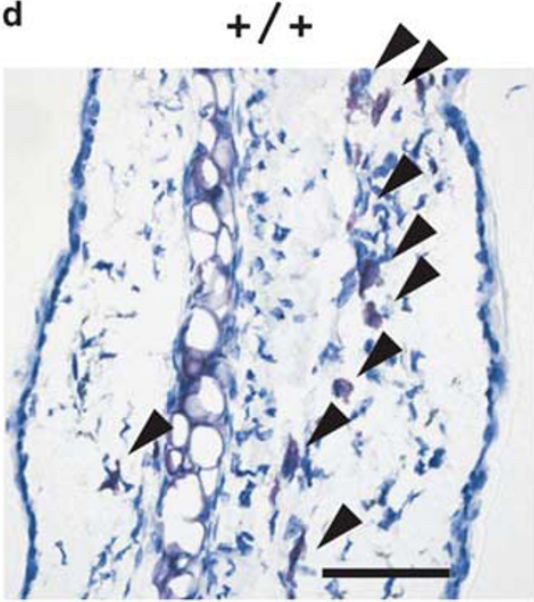

- / -

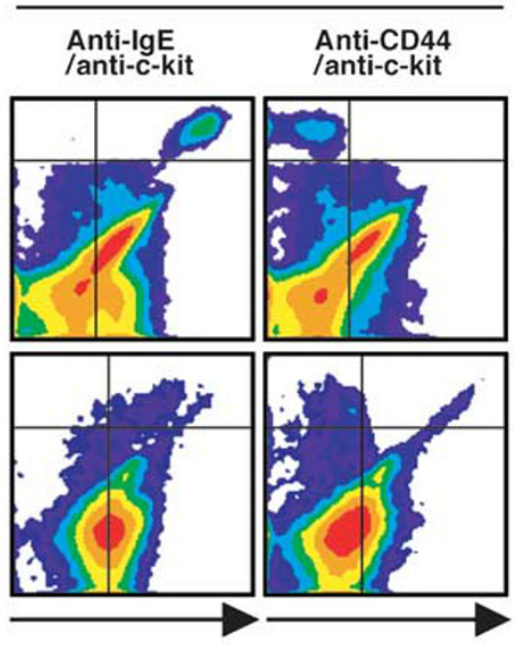

$\mathrm{Fc}_{\varepsilon} \mathrm{R} \mathbf{I}$

CD44

e

- / -
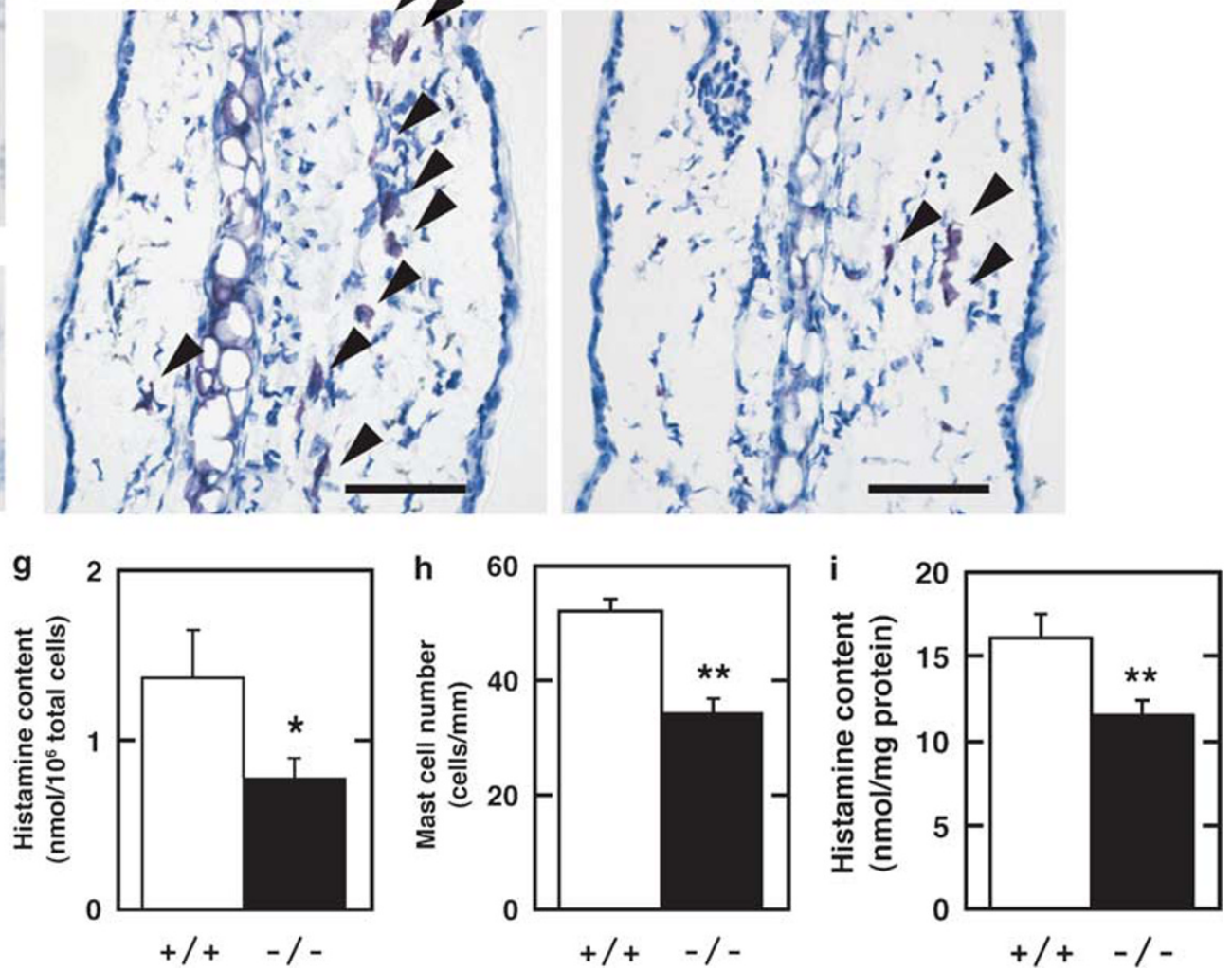

Figure 4 Decrease in the number of mast cells in the $\mathrm{CD}_{4} 4^{-1-}$ mice. (a) Surface expression levels of Fc\&Rl, c-kit, and CD44 in the peritoneal cells, and the cells prepared from the ear tissues of the $\mathrm{CD}_{4} 4^{+/+}$or $\mathrm{CD} 44^{-/-}$mice were measured by flow cytometry. (b-e) The peritoneal cells (b and $\left.\mathbf{c}\right)$ and ear tissues (d and $\mathbf{e}$ ) of the $\mathrm{CD}_{4} 4^{+/+}$(b and $\left.\mathbf{d}\right)$ and $\mathrm{CD}_{4}{ }^{-/-}$(c and $\left.\mathbf{e}\right)$ mice were stained with acidic toluidine blue. Bar $=10 \mu \mathrm{m}(\mathbf{b}$ and $\mathbf{c})$ or $100 \mu \mathrm{m}(\mathbf{d}$ and $\mathbf{e})$. (d) The ear mast cells are indicated by the arrowheads. (f) The percentages of mast cells in $C D 44^{+/+}$and $C D 44^{-/-}$peritoneal cells are presented $(n=5)$. (g) Histamine content in the peritoneal cells of the $C D 44^{+/+}(n=11)$ and $C D 44^{-1-}(n=13)$ mice was measured. (h) The number of mast cells in the ear tissues of $C D 44^{+/+}$and $C D 44^{-/-}$mice was measured $(n=5)$. (i) Histamine content in the ear tissues of $C D 44^{+/+}(n=11)$ and $C D 44^{-/-}(n=13)$ mice was measured. Values are presented as the means \pm s.e.m. ${ }^{*} P<0.05$ and ${ }^{*} P<0.01\left(v s\right.$ CD $44^{+/+}$) are regarded as significant.

(Figure 5d); the number of mast cells in the ear tissues transplanted with $C D 44^{+/+}$BMMCs was approximately twice as large as that in the tissues transplanted with $C D 44^{-1}$
BMMCs at 10 weeks after the injection. The histamine content in ear tissues at 10 weeks after the initial injection reflected the difference in the mast cell numbers (Figure 5e). 
a
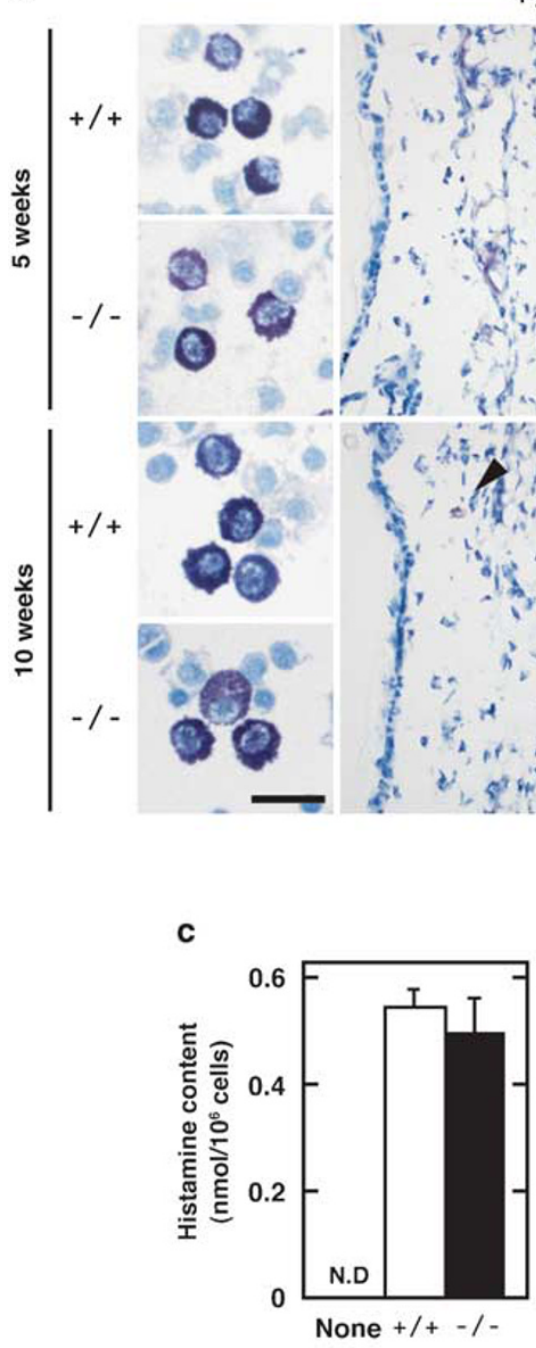

$+1+$

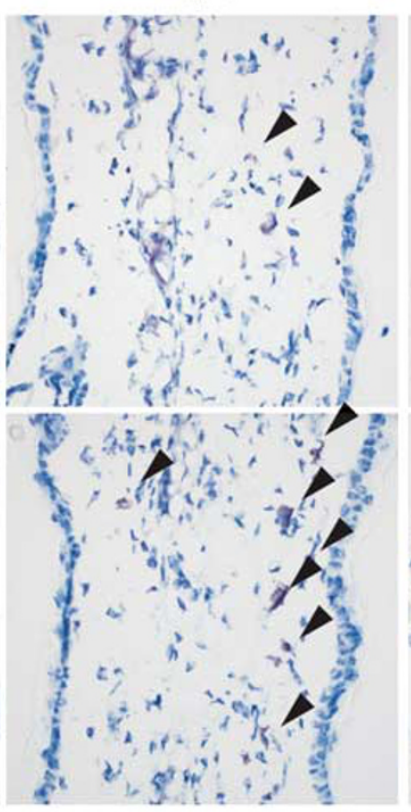

d
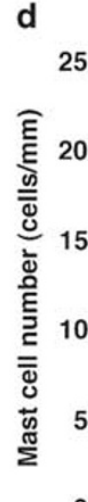

$-1-$

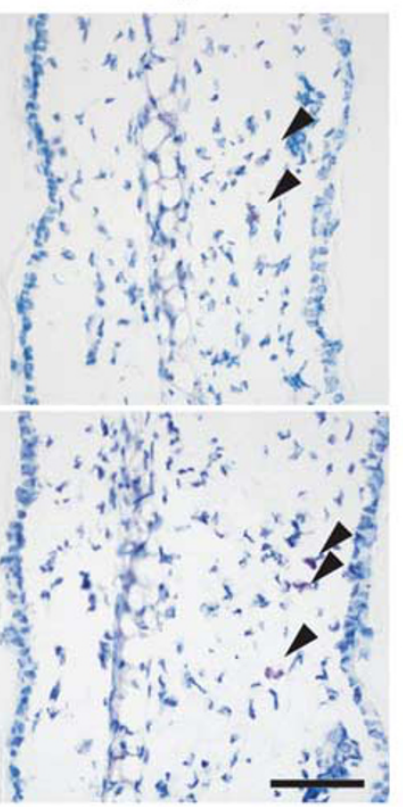

b

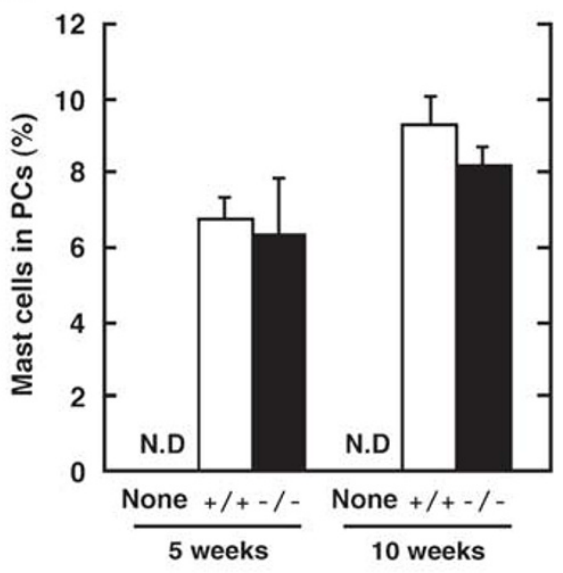

\section{e}

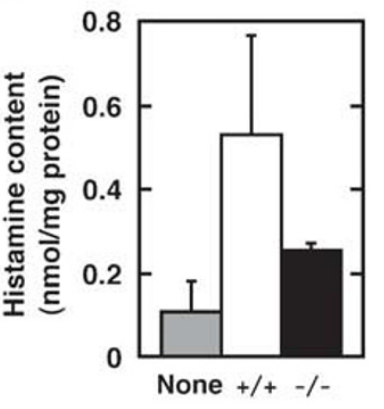

Figure 5 Impaired growth of transplanted $C D 44^{-/-}$mast cells in cutaneous tissues of the mast cell-deficient mice. BMMCs derived from the $C D 44^{+/+}$or

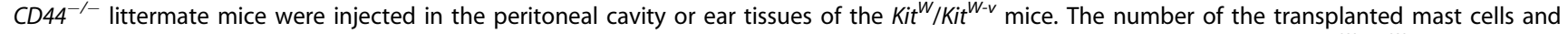
histamine content were measured 5 or 10 weeks after the initial transplantation. (a) The peritoneal cells and ear tissues of the Kit ${ }^{W / K i t}{ }^{W-v}$ mice transplanted with $C D 44^{+/+}(+/+)$and $C D 44^{-/-}(-/-)$BMMCs were stained with acidic Toluidine blue. Bar $=20 \mu \mathrm{m}$ (peritoneal cells) or $100 \mu \mathrm{m}$ (ear tissues). The mast cells in the ear tissues are indicated by the arrowheads. (b) The percentages of mast cells in the peritoneal cells recovered from the Kit ${ }^{W} / K_{i t}^{W-v}$ mice transplanted without (None, $n=3)$ or with the $C D 44^{+/+}(+/+, 5$ weeks; $n=7,10$ weeks; $n=8)$ or CD44 ${ }^{-/-}(-/-, n=5)$ BMMCs were presented. (c) Histamine contents in the peritoneal cells recovered from the $K i t^{W} / K i t^{W-v}$ mice transplanted without (None, $\left.n=3\right)$ or with $C D 44^{+/+}(+/+, n=8)$ or $C D 44^{-1-}(-/-, n=5)$ BMMCs 10 weeks after the initial injection are presented. ND, not detectable. (d) The numbers of mast cells in the ear tissues from the $\mathrm{Kit}^{W} / \mathrm{Kit}^{\mathrm{W-v}}$ mice transplanted without (None, 5 weeks; $n=3,10$ weeks; $\left.n=4\right)$ or with $C D 44^{+/+}(+/+, 5$ weeks; $n=6,10$ weeks; $n=5)$ or $C D 44^{-/-}(-/-$, 5 weeks; $n=7,10$ weeks; $n=3$ ) BMMCs are presented. Values of ${ }^{*} P<0.01$ are regarded as significant. NS, not significant. (e) Histamine contents in the ear tissues recovered from the $\mathrm{Kit}^{W} / \mathrm{Kit}^{\mathrm{W}-\mathrm{v}}$ mice transplanted without (None, $\left.n=4\right)$ or with $\mathrm{CD} 44^{+/+}(+/+, n=5)$ or CD44 ${ }^{-/-}(-/-, n=3)$ BMMCs 10 weeks after the initial injection are presented. All values are presented as means \pm s.e.m.

As the number of the transplanted mast cells in the spleen was found to be very small and similar between the mice injected with $C D 44^{+/+}$BMMCs and those with $C D 44^{-/-}$ BMMCs (data not shown), it is less likely that the absence of CD44 affect the process of mast cell evasion from the injection sites. These results suggest that CD44 is involved in the regulation of mast cell number in cutaneous tissues.

\section{DISCUSSION}

Regulation of Tissue Mast Cell Number by CD44 Expressed in the Mast Cells

Recent studies identified the candidates for the committed mast cell progenitor, although the nature of them remains controversial. $^{19,20}$ As the mast cell progenitor is a rare population as compared with a large number of tissue 
resident mast cells, proliferation after tissue infiltration of immature mast cells should be essential for maintenance of local mast cell number. Although proliferation of mast cells under emergency conditions, such as acute parasite infection, has been investigated in detail, it remains largely unknown how mast cell proliferation is regulated under steady-state conditions. ${ }^{21}$ Our results obtained in the culture system indicated that the upregulation of CD44 during mast cell maturation should promote the proliferation of mast cells. The positive role of CD44 in mast cell proliferation was also supported by two in vivo findings, decrease in the number of tissue mast cells in the $C D 44^{-/}$ mice and the unchanged number of metachromatic cells in cutaneous tissues reconstituted with the $C D 44^{-/-}$BMMCs. As these in vivo data were obtained by the end point measurement, we cannot exclude possibilities that CD44 is involved in the process of maturation and/or survival of cutaneous mast cells. However, involvement of CD44 in maturation and survival of mast cells was not supported by the results obtained in the culture system. Our results collectively suggest that CD44 plays a crucial role in the regulation of mast cell number in the cutaneous tissues.

As accumulating evidence has indicated that CTMCs are involved in a diverse array of immune responses, it is possible that inflammatory responses in cutaneous tissues are affected by the decrease in mast cell number in the $\mathrm{CD} 44^{-/-}$mice. CD44 is a potential therapeutic target for prevention of mast cells hyperplasia, which is often observed in chronic inflammatory diseases, such as atopic dermatitis and rheumatoid arthritis (RA). However, we could not detect significant changes in IgE-mediated immediate allergic responses in the $C D 44^{-/-}$mice (data not shown), such as passive cutaneous anaphylaxis reaction, which is solely dependent on cutaneous mast cells, raising the possibility that the mast cell number in the $C D 44^{-1-}$ mice is enough to evoke such responses. We have to focus on the roles of CD44 under chronic inflammatory conditions, although multiple roles of $\mathrm{CD} 44$, such as extravasation and adhesion of the other leukocytes, would make difficult to ascertain the impact of CD44 expressed in mast cells.

We noticed the inconsistency in the number of peritoneal $C D 44^{-/-}$mast cells; as compared with the CD44 ${ }^{+/+}$mice, the $C D 44^{-1-}$ mice had a slightly but significantly fewer mast cells in the peritoneal cavity, whereas no significant differences were found between the number of transplanted $\mathrm{CD} 44^{+/+}$and $\mathrm{CD} 44^{-/-}$mast cells. This inconsistency may arise from the moderate increase in the number of the transplanted BMMCs in the peritoneal cavity in comparison with that in the cutaneous tissues. It is also possible that the degree of contribution of CD44 is lower in the peritoneal cavity than in cutaneous tissues as the extracellular matrix containing HA is not so much developed in the peritoneal cavity as in cutaneous tissues.

\section{Regulation of CD44 by Tissue Environmental Factors}

Alteration and breakdown of the extracellular matrix is a general feature of chronic inflammatory diseases. Recent studies have indicated that CTMCs are involved in inflammatory arthritis; mast cell-deficient mouse strains were resistant to autoimmune inflammatory arthritis; ${ }^{22}$ and an increase in the local mast cell number is observed in RA and is closely correlated with the clinical progress of this disease. ${ }^{23}$ The increase of mast cells in RA is attributed to the altered connection between mast cells and the extracellular matrix, as the synovial matrix in RA was often disrupted through altered metabolism of HA. ${ }^{24}$ Human $\mathrm{CD} 34^{+}$cell-derived cultured mast cells were reported to bind to HA through CD44 and to shed CD44 molecules upon activation of FceRI, although it remained unknown what is the physiological roles of CD44 in human mast cells. ${ }^{25}$ Shedding of CD44 may affect the fate of tissue mast cells that are activated upon $\mathrm{Fc}_{\varepsilon} \mathrm{RI}$ cross-linking. It is interesting to investigate the roles of CD44 in mast cells under chronic inflammatory conditions, where tissue mast cells are activated.

\section{Mechanism Underlying CD44-Mediated Proliferation}

Accumulating evidence has suggested that CD44 is involved in the proliferation of many cell types, although the underlying molecular mechanism remains largely unknown. Earlier studies indicated that $\mathrm{CD} 44$ can function as a co-receptor to activate the signaling pathway through interacting with several growth factor receptors. ${ }^{26-28}$ It is possible that CD44 functions as a co-receptor to potentiate the growth signaling in mast cells. As c-kit is the dominant receptor that regulates proliferation and differentiation of mast cells, c-kit is one of the possible partners of CD44 when it functions as a co-receptor. Although we could not detect any interaction between CD44 and c-kit by immunoprecipitation (data not shown), further analyses are required about potential crosstalk of the signaling pathway between CD44 and c-kit.

CD44 was reported to modulate cytokine synthesis by leukocytes, such as $\mathrm{T}$ cells and macrophages, ${ }^{7}$ raising a possibility that cytokine synthesis in mast cells are also modulated by CD44. An array of studies showed that mast cells are the sources of cytokines, which can affect their own growth in an autocrine manner. ${ }^{21} \mathrm{CD} 44$ and the extracellular matrix containing HA can trap and concentrate the growth factors and enzymes involved in the cell growth. ${ }^{6}$ In-depth analyses of the matrix components may shed light on the roles of growth factors in mast cell proliferation in the clusters.

Tolg et $a l^{29}$ showed that the receptor for HA-mediated motility (RHAMM), which is known as another HA receptor, functions as an essential regulator of the CD44-ERK1/2 axis in motogenic signaling in fibroblasts. Furthermore, Nedvetzki $e a^{30}$ showed that collagen-induced arthritis was exacerbated in the $C D 44^{-/}$mice, and suggested that the signaling through RHAMM augmented by accumulated HA is responsible for the aggravation of joint inflammation. These findings indicate the co-operative and compensatory 
functions of RHAMM. However, our attempts to show a possible involvement of RHAMM in the regulation of mast cell proliferation have been currently unsuccessful; no significant changes of proliferation were found in the presence of an anti-RHAMM antibody, although the surface expression of RHAMM was detected by flow cytometry (data not shown).

In summary, our results suggest that the number of mature mast cells in cutaneous tissues is regulated at least, in part, by CD44 in mice; CD44 promotes proliferation during the terminal differentiation of tissue mast cells. CD44 might be one of the potential therapeutic targets for chronic cutaneous inflammatory diseases accompanied by mast cell hyperplasia.

Supplementary Information accompanies the paper on the Laboratory Investigation website (http://www.laboratoryinvestigation.org)

\section{ACKNOWLEDGEMENTS}

The authors are grateful to Professor M Miyasaka (Osaka University) for the generous gifts of the series of monoclonal antibody raised against CD44. H Takanao was supported by 21st Century COE Program 'Knowledge Information Infrastructure for Genome Science.' S Tanaka was supported in part by a grant from Uehara Memorial Foundation, Naito Foundation, Kao Foundation for Arts and Sciences, Sankyo Foundation of Life Science, and the Fugaku Trust for Medical Research. K Nakayama, Y Sugimoto, and S Tanaka were supported by Grants-in-Aid for Scientific Research from the Ministry of Education, Culture, Science, Sports and Technology of Japan and from the Ministry of Health and Labor of Japan.

1. Metz M, Grimbaldeston MA, Nakae $S$, et al. Mast cells in the promotion and limitation of chronic inflammation. Immunol Rev 2007;217: 304-328.

2. Metz M, Maurer M. Mast cells-key effector cells in immune responses. Trends Immunol 2007;28:234-241.

3. Kitamura Y. Heterogeneity of mast cells and phenotypic change between subpopulations. Annu Rev Immunol 1989;7:59-76.

4. Takano $\mathrm{H}$, Nakazawa S, Okuno $\mathrm{Y}$, et al. Establishment of the culture model system that reflects the process of terminal differentiation of connective tissue-type mast cells. FEBS Lett 2008;582:1444-1450.

5. Ponta $H$, Sherman L, Herrlich PA. CD44: from adhesion molecules to signalling regulators. Nat Rev Mol Cell Biol 2004;4:33-45.

6. Puré E, Cuff CA. A crucial role for CD44 in inflammation. Trends Mol Med 2001;7:213-221.

7. Tammi M, Day AJ, Turley EA. Hyaluronan and homeostasis: a balancing act. J Biol Chem 2002;277:4581-4584.

8. Laurent TC, Fraser JR. Hyaluronan. FASEB J 1992;6:2397-2404.

9. McLachlan JB, Hart JP, Pizzo SV, et al. Mast cell-derived tumor necrosis factor induces hypertrophy of draining lymph nodes during infection. Nat Immunol 2003:4:1199-1205.
10. Jawdat DM, Rowden G, Marshall JS. Mast cells have a pivotal role in TNF-independent lymph node hypertrophy and the mobilization of Langerhans cells in response to bacterial peptidoglycan. J Immunol 2006;177:1755-1762.

11. Lu LF, Lind EF, Bennett DC, et al. Mast cells are essential intermediaries in regulatory T-cell tolerance. Nature 2006;442:997-1002.

12. Protin U, Schweighoffer T, Jochum W, et al. CD44-deficient mice develop normally with changes in subpopulations and recirculation of lymphocytes subsets. J Immunol 1999;163:4917-4923.

13. Tanaka S, Takasu Y, Mikura S, et al. Antigen-independent induction of histamine synthesis by immunoglobulin $\mathrm{E}$ in mouse bone marrowderived mast cells. J Exp Med 2002;196:229-235.

14. He D, Esquenazi-Behar S, Soter NA, et al. Mast-cell heterogeneity: functional comparison of purified mouse cutaneous and peritoneal mast cells. J Invest Dermatol 1990;95:178-185.

15. Tanaka S, Mikura S, Hashimoto $\mathrm{E}$, et al. $\mathrm{Ca}^{2+}$ influx-mediated histamine synthesis and IL-6 release in mast cells activated by monomeric IgE. Eur J Immunol 2005;35:460-468.

16. Kitchen JR, Cysyk RL. Synthesis and release of hyaluronic acid by Swiss 3T3 fibroblasts. Biochem J 1995;309:649-655.

17. Bergstresser PR, Tigelaar RE, Tharp MD. Conjugated avidin identifies cutaneous rodent and human mast cells. J Invest Dermatol 1984;83:214-218.

18. Zheng Z, Katoh $S$, He Q, et al. Monoclonal antibodies to CD44 and their influence on hyaluronan recognition. J Cell Biol 1995;130: 485-495.

19. Chen $C$, Grimbaldeston MA, Tsai M, et al. Identification of mast cell progenitors in adult mice. Proc Natl Acad Sci USA 2005;102: 11408-11413.

20. Arinobu Y, Iwasaki H, Gurish MF, et al. Development checkpoints of the basophil/mast cell lineages in adult murine hematopoiesis. Proc Natl Acad Sci USA 2005;102:18105-18110.

21. Metcalfe DD, Baram D, Mekori YA. Mast cells. Physiol Rev 1997;77:1033-1079.

22. Lee DM, Friend DS, Gurish MF, et al. Mast cell: a cellular link between autoantibodies and inflammatory arthritis. Science 2002;297: 1689-1692.

23. Maruotti N, Crivellato E, Cantatore FP, et al. Mast cells in rheumatoid arthritis. Clin Rheumatol 2007;26:1-4.

24. Naor D, Nedvetzki S. CD44 in rheumatoid arthritis. Arthritis Res Ther 2003;5:105-115.

25. Fukui $M$, Whittlesey $K$, Metcalfe DD, et al. Human mast cells express the hyaluronic-acid-binding isoform of CD44 and adhere to hyaluronic acid. Clin Immunol 2000;94:173-178.

26. Sherman LS, Rizvi TA, Karyala S, et al. CD44 enhances neuregulin signaling by Schwann cells. J Cell Biol 2000;150:1071-1084.

27. Singleton PA, Salgia R, Moreno-Vinasco L, et al. CD44 regulates hepatocyte growth factor-mediated vascular integrity. J Bio Chem 2007;282:30643-30657.

28. Ghatak S, Misra S, Toole BP. Hyaluronan constitutively regulates ErbB2 phosphorylation and signaling complex formation in carcinoma cells. J Biol Chem 2005;280:8875-8883.

29. Tolg C, Hamilton SR, Nakrieko KA, et al. Rhamm-/- fibroblasts are defective in CD44-mediated ERK1, 2 motogenic signaling, leading to defective skin wound repair. J Cell Biol 2006;175:1017-1028.

30. Nedvetzki S, Gonen E, Assayag N, et al. RHAMM, receptor for hyaluronan-mediated motility, compensate for CD44 in inflamed CD44-knockout mice: a different interpretation of redundancy. Proc Natl Acad Sci USA 2004;101:18081-18086. 